\title{
The ROTSE Detection of Early Optical Light from GRB 990123
}

\author{
Galen Gisler*
}

On behalf of the ROTSE collaboration: \begin{abstract}
John J. Szymanski*, and James A. Wren*
\author{
${ }^{*}$ Los Alamos National Laboratory ${ }^{1}$ \\ ${ }^{\dagger}$ University of Michigan
}

${ }^{\dagger}$ Lawrence Livermore National Laboratory
\end{abstract}

Carl W. Akerlof ${ }^{\dagger}$, Richard J. Balsano*, Jeffrey J. Bloch*, Donald E. Casperson*, Sandra J. Fletcher*, Galen R. Gisler*, Jack G. Hills*, Robert L. Kehoe ${ }^{\dagger}$, Brian C. Lee ${ }^{\dagger}$, Stuart L. Marshall ${ }^{\ddagger}$, Timothy A. McKay ${ }^{\dagger}$, Richard S. Miller*, William C. Priedhorsky*,

It is perhaps odd to present a paper on a ground-based observational astronomy project at a workshop on small satellites, but the Robotic Optical Transient Search Experiment (ROTSE) is indeed a small project (if not a small satellite), and we have recently obtained a result of high interest to the small-satellite community. This result was the detection of very early time light from a gamma-ray burst, at a brightness accessible to very small telescopes. We argue that this result should influence the design of future gamma-ray burst missions, both on the ground and in space. The details of our observation of GRB 990123 have been published, subsequent to this workshop, in the journal Nature (Akerlof et al. 1999), and I therefore present in this paper an anecdotal review of gamma-ray bursts, our system, this particular event, and subsequent developments in our project.

Gamma-Ray Bursts (GRBs) were discovered in the early 1970's by the Los Alamos series of Vela satellites, which had been looking for violations of the limited nuclear test-ban treaty. Many satellite missions since those early days have studied them, but they remain among the most puzzling phenomena in the cosmos. By far the most prolific source of data on GRBs has come from the Compton Gamma-Ray Observatory, primarily the Burst and Transient Source Experiment (BATSE) on board that satellite. By this time, many thousands of bursts have been observed, and new ones are discovered at the rate of about one per day. New generations of satellites, including HETE, SWIFT, BALLERINA (all discussed at this meeting) will be addressing these phenomena in the future.

1) email: gisler@lanl.gov

CP499, Small Missions for Energetic Astrophysics, edited by Steven P. Brumby (ㄷ) 1999 American Institute of Physics 1-56396-912-2/99/\$15.00 
Since the beginning of the study of GRBs, attempts have been made to observe these events at other wavelengths, principally using optical telescopes on the ground. Archive searches and photographic patrols yielded no counterparts, and all attempts to perform simultaneous observations were hampered by the poor positional information obtained by the gamma-ray observations, by the short duration of the events, and by the difficulty in disseminating alerts quickly.

Because of the lack of observations at other wavelengths, understanding of these phenomena developed only slowly. Phenomenologically, GRBs are intense, brief, localized emissions of radiation at energies from $10 \mathrm{~s}$ of $\mathrm{keV}$ through $10 \mathrm{~s}$ of $\mathrm{MeV}$, with isolated instances of reported detections at $10 \mathrm{~s}$ of $\mathrm{GeV}$ and even $\mathrm{TeV}$ energies. Their durations range from 10s of milliseconds to hundreds of seconds, with a hint of a division into two populations separating around a few seconds. When they occur, they are by far the brightest objects in the sky at their wavelengths. Because of their high brightness, it has been possible to study their light curves and gamma-ray spectra in great detail. They have an enormous diversity in their light curves, from simple isolated pulses to complex and multiple structures; sometimes their are precursors, sometimes aftershocks, and the spectral energy distributions have been observed to change in a variety of ways during a burst. Unfortunately, though the light curves are well characterized, they don't provide much information to aid understanding. They may not be a homogeneous population, yet they differ profoundly from all other known astrophysical phenomena in being transient, violent, and nonrepetitive. The conservative approach is to treat them as a relatively homogeneous population until we know better.

Population studies of GRBs have led to the conclusion that they are very distant, probahly cosmological. Firstly, they are isotropic to a degree shared by no population of relatively nearby known sources, and secondly, the number-brightness distrihution shows a deficit of faint sources as might bè produced by cosmic evolution.

Theoretical understanding of GRBs has been slow, though there have been hundrexls of papers devoted to the subject, many developing hypotheses that now seem rather quaint. The gradual acceptance of a cosmological distance scale for GRBs has limited the playing field to those theories that can provide the prodigious energies that are required. Collisions of neutron stars with each other, or with black holes, or processes leading to the rapid formation of a black hole, such as special kinds of supernovae, or hypernovae, seem to be among the leading candidates, though the processes by which much of this energy is liberated in the form of gamma-rays remains tentative and speculative.

Alove all, we've needed more information on these objects, particularly at differcut wavelengths, but that has been very hard to come by. Without good positions for CiRBs, they cannot be identified with other known types of sources. But it is rlifficult to get good positions from the gamma-rays alone. Our detectors are not sufficiently discriminatory in direction. Our main tool for getting directions las bxen the use of timing and intensity ratios among different detectors, or elements of a single detectors. The BATSE detectors provide error circles of degrees 
in this way, but only with interplanetary baselines is this technique good enough to provide error circles of arc minutes, enabling identifications. Until 1997, the Interplanetary Network (IPN), involving several satellites at different locations in the solar system, was the only source of good GRB positions. However, IPN positions are never available until hours (if not days or weeks) after the burst occurs, prohibiting simultaneous observations.

The lack of good rapid positions led to the development of wide-field rapid response optical telescope experiments and a network of rapid alert dissemination via the internet, first known as Bacodine (BATSE Coordinate Distribution Network) and then as GCN (Gamma-ray Coordinates Network). Among the optical experiments that were deployed and subscribing to GCN were the Explosive Transient Camera (ETC, Krimm et al 1996), the Gamma-Ray Optical Counterpart Search Experiment (GROCSE, Lee et al 1997), the Livermore Optical Transient Imaging System (LOTIS, Park et al 1997), and our own system, ROTSE. Collectively, these wide-field experiments ran for a dozen years without seeing any optical counterparts to GRBs.

The strategy of the wide field search experiments was essentially to have a small automated telescope in a constant state of readiness (generally performing background observations); it receives coordinates of a GRB very quickly from GCN, and if the object is in an accessible part of the sky, it slews to the indicated position and performs a sequence of exposures, possibly tiling to look at an even wider area of sky. Afterwards, the entire exposed field is searched for a transient or variable source. This approach was mostly unsuccessful, though useful limits were placed on a few GRB optical counterparts.

Our ROTSE projected consisted of two separate systems, ROTSE I, a 2x2 array of Canon telephoto lenses (f/1.8, 200mm focal length) with large format CCD imagers (Thomson $14 \mathrm{~m} 2048 \times 2048$ pixels), and ROTSE II, a $0.45 \mathrm{~m}, \mathrm{f} / 1.9$ telescope. ROTSE I has a combined field of view of 16.5 degrees, well suited to covering a large fraction of the BATSE error circle. It has been rumning in automated mode since March 1998, and has so far accumulated more than 2 Terabytes of data in its background mode of continuously patrolling the overhead night sky. It has responded to a few dozen alerts, with an average response time of about 10 seconds from the BATSE trigger time (including an average of 3 seconds slewing time). ROTSE II has a field of view of just under 2 degrees, and is not yet working automatically.

The major breakthrough in GRB studies occurred in 1997 with the first detection of an optical afterglow of a gamma-ray burst, but this was not accomplished by any of the wide-fiekd searches. Other teams of optical and radio observers had subscribed to GCN with the hope of seeing afterglows of GRBs with conventional narrow-field large-aperture telescopes. They succeeded in discovering the first optical counterpart when a source of accurate positions for GRBs became available. This source was the Italian-Dutch Beppo-SAX satellite, which had a GRB monitor plus a wide-field $\mathrm{X}$-ray camera. The rough positions given by the monitor were sufficient to point the X-ray camera, which in turn gave positions to a few minutes 


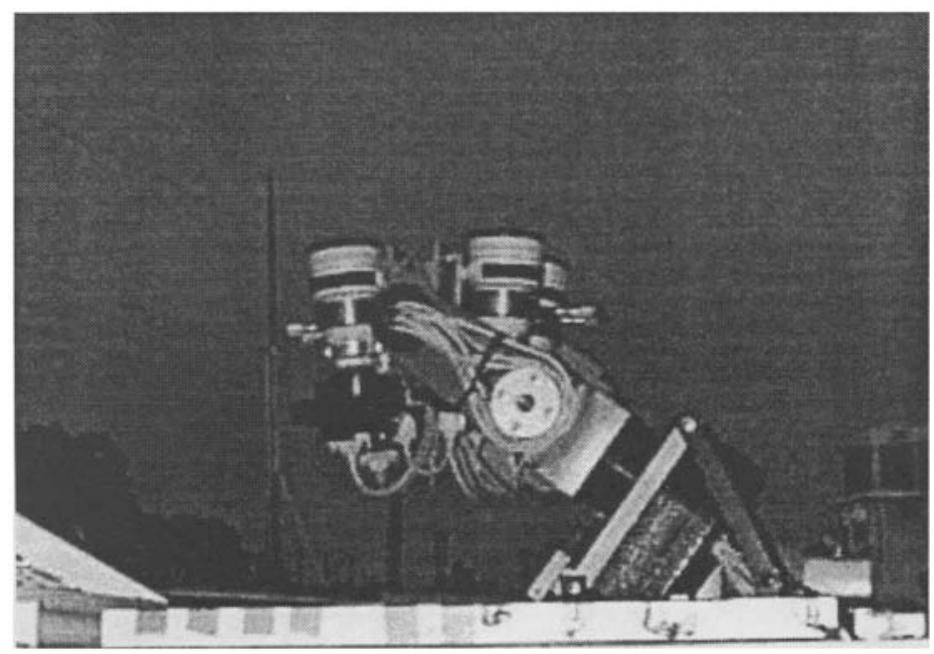

FIGURE 1. ROTSE I atop its enclosure, pointed at zenith

of arc. Because this satellite was not in constant communication with the ground, and because the repointing took some time, the accurate positions were not available until several hours after the burst occurred. But this was significantly more rapid than the IPN positions, which were of similar accuracy.

The first two optical counterparts found, GRB 970207 and GRB 970508, by van Paradijs, Kulkarni, Frail, and others, using the Beppo-Sax positions, proved a sensational boost to this field. We finally had direct confirmation, via the redshift of 970508 , that at least some GRBs (and probably all) were at cosmological distances. The study of afterglows became an industry in itself, with more observers signing up to perform counterpart observations with their large telescopes. A dozen optical afterglows were discovered over the next two years, with some radio afterglows as well, and redshifts were measured for roughly half. Several common features were noted, including power-law decays, indicating deceleration by an external medium. All were in host galaxies, though not centrally located, and those with measured redshifts were all at distances greater than $z \sim 0.8$ (except for one, GRB 980425, which was associated with a supernova in a relatively nearby galaxy). One burst, GRB 971214, holds the record for the most distant, at $z=3.4$. With actual measurements of distances, and radiation in new wavelength regimes, came new impetus for theoretical developments. The energy requirements were now accepted to be greatly in excess of supernova energies, and theoretical models now looked toward black hole formation scenarios. Additionally, the fireball models became more sophisticated and interesting, involving external shocks and internal shocks 


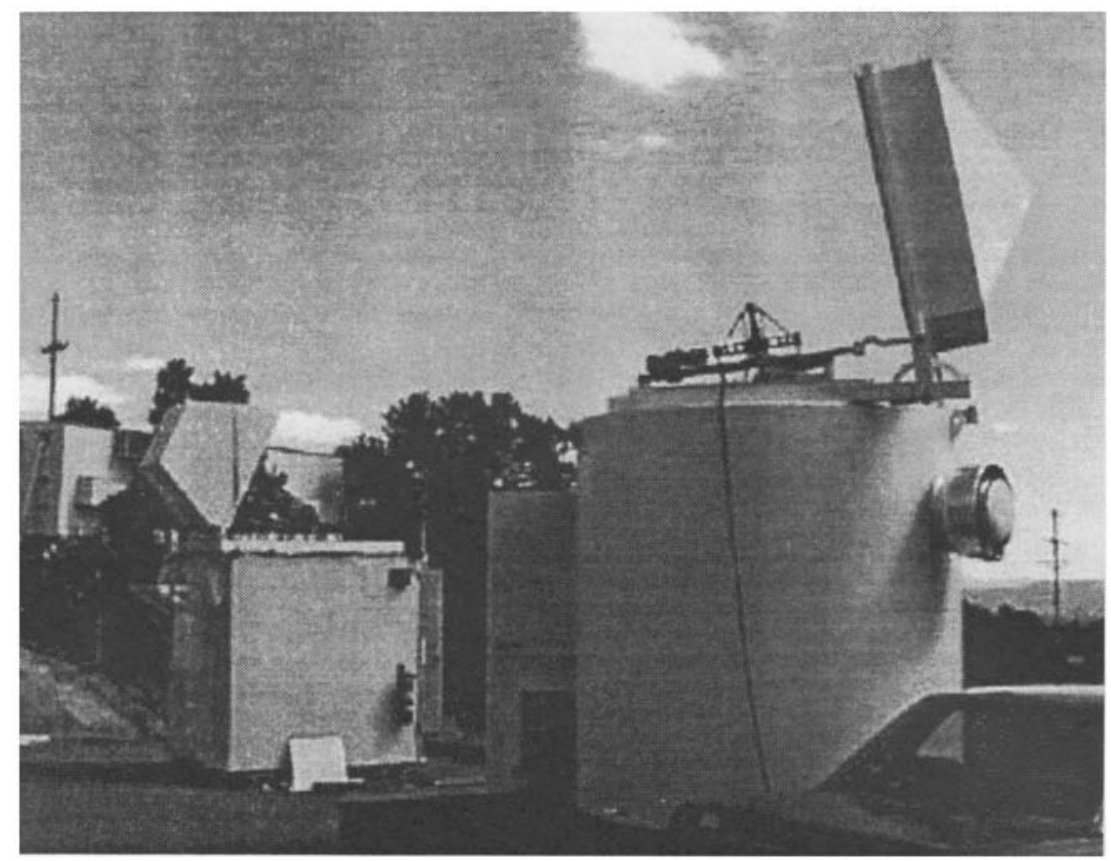

FIGURE 2. ROTSE I (left) and ROTSE II with clamshells partially open.

as well as a central cataclysmic source. Comparisons of time structures in the optical and radio led to new insights, and radio scintillation observations gave the first measurements of the expansion of the fireball.

Unfortunately, it also seemed clear that large telescopes were indeed needed to play in this game, because the afterglows themselves were very faint. In particular, 970508 had apparently been caught on the rise, peaking at 19th magnitude. Things were looking very bad for wide-field early-time searches for gamma-ray optical counterparts.

In ROTSE, we worked on placing early limits on some bursts, and looked toward changing our observing strategies to achieve the depth that appeared necessary to find the late-time afterglows. This involved longer exposures, less tiling, sacrificing coverage of the extended error field, etc. A delicate problem we faced was: what if we found a very bright and rapidly fading transient, at early times, somewhere in the 256 square degrees covered by ROTSE I. Would anyone believe us? Though we were looking in a unique region of parameter space, we were ourselves doubtful.

Then Nature presented us with a gift. On 1999 January 23, 09:47 UT (= 02:47 MST) BATSE and Beppo-SAX both triggered on a strong GRB. Four seconds later, the GCN signal arrived at ROTSE I, which terminated its sky patrol exposure 
and began steering toward the early BATSE coordinates (which were 9 degrees away from the subsequent localization). By ten seconds after the burst trigger, ROTSE I had begun its first 5-second exposure on the target. Unfortunately, this exposure, and all subsequent odd-numbered exposures, were lost due to software errors. The second exposure, and the first one saved, was begun 22 seconds after the BATSE trigger, recording a transient stellar object at 12th magnitude. The next saved exposure, at 47 scconds after the trigger, recorded the same transient at 9 th magnitude. Since this burst was a long burst, this and two other optical frames were obtained while the gamma-rays were still being received by BATSE. Jim Wren, who was covering shift that Saturday morning, was awakened by his pager, which reported that ROTSE I was responding to a BATSE alert. He verified that the system was working, and went back to sleep. By 45 minutes after the initial trigger, the ROTSE I alert response was finished, and the system resumed the interrupted sky patrol. Our transient detection was in our data, but as yet undiscovered.

Four hours after the grigger, Beppo-SAX reported a position for the burst that was correct to 5 minutes of arc (Piro et al GCN Circ. No. 199). Immediatcly, Odewahn, Bloom and Kulkarni used a 60 -inch telescope to point to that position, and identified an optical transient at 18th magnitude, which they promptly reported (Odewahn et al GCN Circ. No. 201). Eight hours after the trigger, Carl Akerlof and Tim McKay of our collaboration used the Beppo-SAX position to search our data, and they found the transient at the same position reported by Odewahn et al, but much brighter. This was likewise promptly reported (Akerlof and McKay GCN Circ. No, 205). The report of the redshift, $\mathrm{z}=1.6$ (Hjorth et al, GCN Circ. No. 219), came a day later, and proved this burst to be the most luminous optical Hash ever seen, and the most violent by far.

The discovery of simultaneous optical emission in a gamma-ray burst at such a brightness was not entirely unexpected. Some theorists had been encouraging early-time searches for several years (e.g. Meszaros and Rees 1997). Nevertheless, it is fair to say that this event has renewed efforts to cover the time domain in astrophysics to a much greatcr extent than was previously conceived.

Unfortunately, the discovery of early bright transients is still not favored by the present suite of detectors. ROTSE I needed both the early BATSE position to point the telescope, and the subsequent Beppo-SAX localization to find the 990123 transient in the wide field. Because the early BATSE position was off by 9 degrees, we very nearly missed it! Future satellites will give more accurate early positions, but we must stress the importance of getting those positions to the ground as rapidly as possible. Some proposed future satellites will have co-mounted optical telescopes, but because of the necessity of conserving angular momentum while slewing the telescopc, rapid response from such a system is very difficult to achieve. Ground-based instruments can slew to a position much more rapidly, easily compensating for the data processing and transmission time. In addition, ground-based instruments are much cheaper and easier to maintain that optical telescopes in space.

We are presently attempting to expand our system to include several additional 

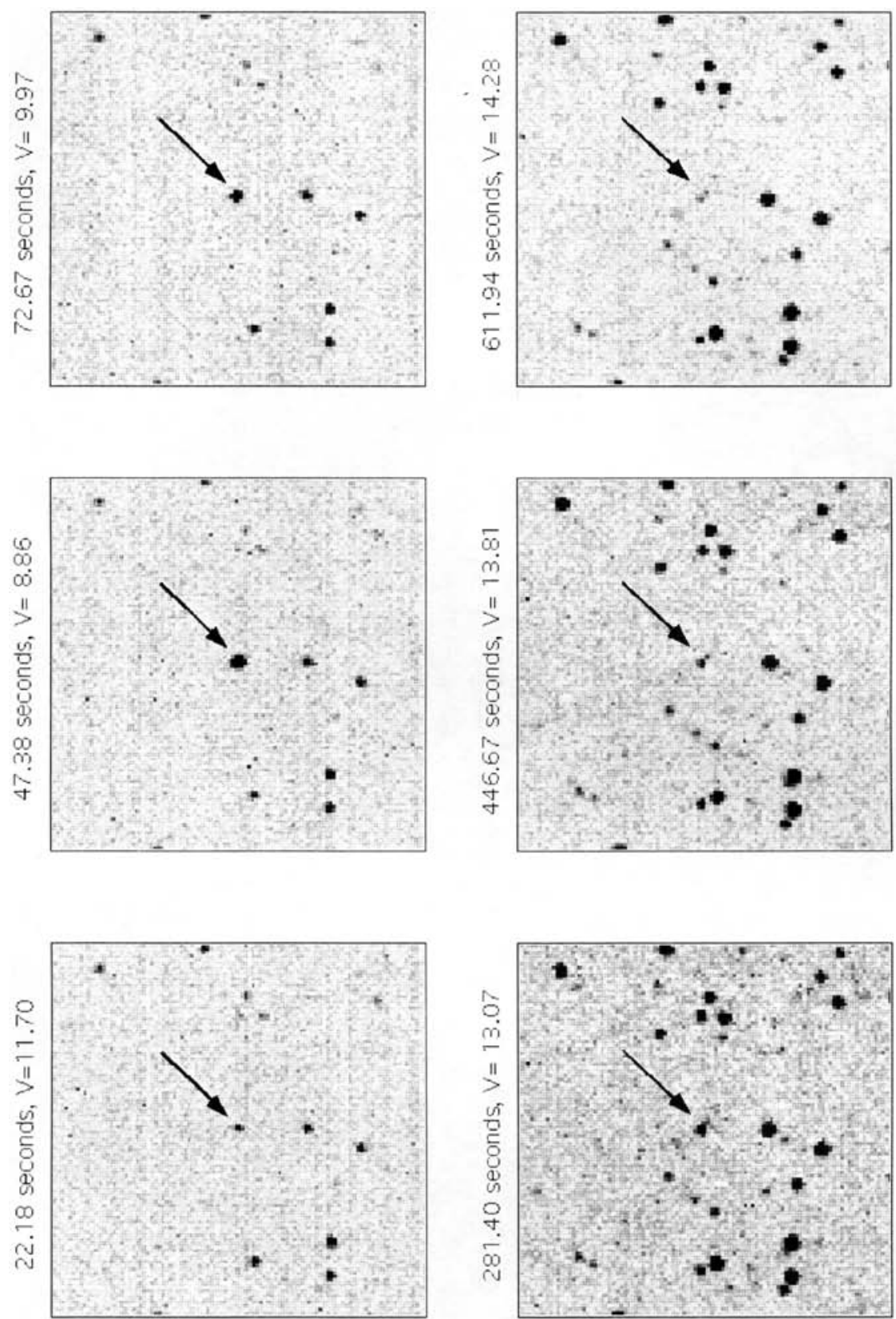

FIGURE 3. The six ROTSE frames for the transient associated with GRB 990123 
sites around the world, widely separated in longitude and latitude, so that we may be in a very good position to respond to alerts from the future generation of satellites (HETE II, SWIFT, GLAST).

\section{REFERENCES}

1. C. Akerlof, R. Balsano, S. Barthelmy, J. Bloch, P. Butterworth, D. Casperson, T. Cline, S. Fletcher, F. Frontera, G. Gisler, J. Heise, J. Hills, R. Kehoe, B. Lee, S. Marshall, T. Mckay, R. Miller, L. Piro, W. Priedhorsky, J. Szymanski \& J. Wren, "Observation of contemporaneous optical radiation from a gamma-ray burst", Nature 398, 400 (1999).

2. C.W. Akerlof \& T.A. McKay, GCN Circ. No. 205 (1999).

3. J. Hjorth et al, GCN Circ. No. 219 (1999).

4. H.A. Krimm, R.K. Vanderspek, \& G.R. Ricker, "Searches for optical counterparts of BATSE gamma-ray bursts with the Explosive Transient Camera", Astron. Astrophys. Suppl. 120, 251 (1996).

5. B. Lee et al, "Results from Gamma-Ray Optical Counterpart Search Experiment, a real-time search for gamma-ray burst optical counterparts", Ap.J. 482, L125 (1997).

6. S.C. Odewahn et al, GCN Circ. No. 201 (1999).

7. P. Meszaros \& M. Rees, "Optical and long-wavelength afterglow from gamma-ray bursts", Ap. J. 476, 232 (1997).

8. H.S. Park et al, "New constraints on simultaneous optical emission from gamma-ray bursts measured by the Livermore Optical Transient Imaging System experiment", Ap. J. 490, L21 (1997).

9. L. Piro et al, GCN Circ. No. 199 (1999). 